\title{
DESIGNING DISTRIBUTED TASK PERFORMANCE IN SAFETY-CRITICAL SYSTEMS EQUIPPED WITH MOBILE DEVICES
}

\author{
Ana-Maria-Marhan, Fabio Paternò, Carmen Santoro \\ ISTI-CNR, Via G.Moruzzi 1, 56124 Pisa, Italy
}

Abstract: This paper describes a method aiming to support the design of interactivesafety critical systems. The method proposes an original integration of approaches usually considered separately, such as task modelling and distributed cognition. The basic idea is that analysing task performance requires a clear understanding of the information needed to accomplish the task and how to derive such information from both internal cognitive representations and external representations provided by various types of artefacts. We also report on a first application of the method to a case study in the Air Traffic Control (ATC) domain.

Key words: Human-Computer Interaction, Methodologies, Design, Task Models.

\section{INTRODUCTION}

Recent developments in wireless communication, distributed systems, together with increases in the power and interactive capabilities of handheld and portable devices provide users with the possibility of a wide-ranging, continuous access to computing resources in a variety of contexts. One of the controversial issues we intend to address in this paper is whether, and in which way, new mobile technology can offer meaningful support to accomplish critical tasks while preserving safety and usability.

To this end, we have extended the analysis of deviations developed in our previous work (Paternò and Santoro, 2002), and provided more explicit consideration of the distributed cognitive resources supporting task performance. The idea guiding this work is that a criticality, or breakdown in 
task performance is the consequence of an inadequate access to information supporting task accomplishment. By integrating a task-based approach to design and evaluation of interactive systems with a distributed cognition analysis (Hutchins, 1995), we aim to achieve a twofold objective:

- To identify a design method that systematically analyses task accomplishment, detects potential deviations, and provides design criteria grounded on distributed cognition analysis;

- To support designers in analysing the impact of introducing mobile, wireless devices in existing technological contexts, and its potential implications in terms of user support.

In the following sections, we first discuss related work, then we move on to describe the proposed method; further on we will introduce a case study in the area of ATC, and show how the proposed method can be applied considering a specific situation.

\section{RELATED WORK}

While the theoretical framework of distributed cognition seeks to offer a systemic attention on cognition (as embedded in its environmental setting), the main limitation of distributed cognition-based approaches is that it seems to fail to provide designers with systematic support in order to translate some interesting findings and general principles in specific design criteria. Hence, in this paper we attempt to integrate a systematic taskbased approach to design and evaluation with criteria derived from a distribute cognition analysis.

Task-based approaches have long been considered in system design and evaluation. Recently, automatic tool support became available. For instance, CTTE environment provides an integrated set of modelling tools (Mori, Paternò, and Santoro, 2002) that allows designers to analyse particular sequence of tasks and to compare alternative paths available (i.e., when the tasks are carried on in different contexts, or using different computing platforms) in order to attain a specific goal.

Such features are particularly relevant when Air Traffic Control (ATC) domain is considered. ATC is an application with well defined goals (ensuring safe and efficient air traffic flow), while a various flexible ways are available to achieving such goals. Complex flows of information can be identified within the interaction among a number of actors (controllers, pilots, aerodrome technicians, etc.), and their technical contexts (cockpit, ATC control centres, aprons, etc.), co-operating for the common purpose of ensuring air traffic safety and efficiency. 
Although the flexibility introduced by mobile devices can find interesting applications in this area, little research work has been addressing the problem of mobile devices in safety-critical contexts. Especially PDAs seems to capture both researchers' and industry's interest: Mertz et al (2000), Buisson \& Jestin (2001) present some scenarios that use PDAs for navigating in the ATC information space. A prototype developed at CENA on the basis of DigiStrips (http://www.tls.cena.fr/divisions/PII/digistrips/), addresses the problem of electronic stripping using a PDA platform. However, the presentation of information using a PDA raises some interesting issues, primarily related to the limitations in displaying information on small-screen devices (particularly when it is question of relatively complex information like procedures or rules which, in ATC, can include maps and long structured text).

\section{A METHOD FOR DESIGN AND EVALUATION OF SAFETY-CRITICAL SYSTEMS SUPPORTED BY MOBILE DEVICES}

One starting point for this research has been the deviation analysis (Paternò and Santoro, 2002). This method involves three main steps:

- Development of the task model of the application considered; the purpose is to provide a description logically structured in a hierarchical manner of tasks that have to be performed, including their temporal relationships, objects manipulated and tasks' attributes.

- Analysis of deviations related to the basic tasks, which are tasks that the designer deems should be considered as units.

- Analysis of deviations in high-level tasks, these tasks allow designer to identify group of tasks and consequently analyse deviations that involve more than one basic task (e.g. deviations concerning whether the appropriate tasks are accomplished following a correct ordering).

In this method, the ConcurTaskTrees (CTT) notation has been used (Paterno', 2003) for task modelling. CTT task models are structured in a hierarchical manner that allows an analysis at various levels of abstractions, and support a detailed analysis of tasks, temporal relationships between tasks, and objects manipulated by tasks. CTT objects are classified in perceivable (objects represented at the interface level, that can be directly perceived by the user), cognitive (User internal representations of the informational entities) and domain objects (entities that are internally represented and manipulated by the system). Tasks are classified depending on their performance allocation: user tasks require only an internal 
performance, interactive tasks require interaction between the user and the external world, and system tasks are completely automatic tasks.

While analysis of deviations based on CTT task models has proved to be useful in generating some interesting results, we realised that its effectiveness can be increased in order to identify possible breakdowns in task performance.

\subsection{Integrating CTT task modelling and DC analysis}

This approach is based on the idea that a criticality in user - system interaction is the consequence of an inadequate access to the information distributed among the interacting components. Based on the description of the (idealised or real) plan of tasks the user needs to follow in order to achieve a goal, the analyst will identify the possible configurations of the resources supporting user actions while carrying out such plans. The method we propose allows a pro-active estimation of those aspects that might affect safety following the introduction of new artefacts in the work setting, and the identification of alternative design options.

The basic steps considered are:

- Identifying tasks (the task considered could be basic tasks in the CTT model, or higher level tasks);

- Determining the resources required to perform the task;

- Defining the most dangerous potential safety-critical deviations for each task;

- Based on the questions stimulated by a distributed cognition approach, identify and evaluate new design solutions that imply less safety-critical interactions

- A set of heuristics extracted from the Distributed Cognition literature orients the analyst's exploring the distributed resources represented within the task space, identifying potential breakdowns or failures can occur owing to a specific distribution of resources across the interactive system, and allows him to systematically reason about alternative ways of distribution of resources during task performance and their impact.

\subsection{Analysis steps}

In carrying on the analysis, several aspects need to be carefully identified : role, task, representations, and deviations.

1. Analysis of the task and related properties: this phase has to identify the goal of the task and a number of task properties that could be relevant for analysing whether its current design is appropriate or possible improvements in terms of system safety and usability are needed: 
1.a Task category and type: depending on task category, different types of actions are meaningful (e.g. for an interactive task, possible actions are: monitoring, control, edit, etc.)

1.b Task platforms: it is possible to specify the platforms embedding the resources supporting task performance.

1.c Task frequency: the amount of time the user devotes to the considered activity; it might indicate the overall 'criticality' or 'importance' of the task.

1.d Other properties (that could affect task 'criticality'): need of real time performance/responses, task urgency, strong dependency with other tasks.

2. Analysis of the representations associated with the task - For each task, the supporting representations (and related properties) are identified: 2.a Availability: it evaluates the type of "presence" of a certain representation in the user's context, differentiating between external or internal (i.e., represented at the user's level) representations. Depending on the value of this property, it could be meaningful to evaluate other properties such as:

2.b. Accessibility: type of access user has to a representation. Different types of accessibility (sequential/concurrent) could be exploited for externally available representations. Also, the analysis of visibility might be connected with the type of media/device used.

2.c. Mobility: whether the access to the information requires the user to move or the user can move while accessing information.

2.d Observability: refers to the extent the perception of a representation is: i) Local to individuals; ii) Shared (e.g. by the members of a team); iii) Globally available to all. This property might be connected with the type of supporting platform (for example if controllers annotate a strip on their PDAs, this information will be available locally to them).

2.e Persistence: whether transient or permanent access to information is allowed.

2.fFlexibility of modifying the representation, ability to flexibly update and modify the representation, for example allowing a person to annotate an external representation (i.e. strips).

2.g Operations and actions supported

2.g.1 Comparability: with other objects / representations available in the user's context;

2.g.2 Combinability: allowing users to combine information from different sources;

2.g.3 Ease of production: allowing reconfiguring and multiple views of information; 
3. Analysing deviations from the task plan - In this phase a combined analysis and evaluation of the information gathered in the previous steps has to be carried out. Such analysis has to evaluate if the current configuration of representations (with their own specific properties) is effective for the task considered (and in the way this task is supposed to be carried out in the considered system). For example, if a certain task results to be a frequent monitoring task and in the considered system the representation of the current situation cannot easily be compared with the expected situation (because, e.g. the expected situation is an internal representation or it is an external representation but it does not reflect the controller's mental model), this could put a heavy (and risky) workload on the controller who continuously has to accommodate this information before actually using it. More in detail, our evaluation will be driven by means of a number of guidewords that will be mainly focused on possible 'deviations' occurring on representations with regard to the performance of the considered task, namely:

3.a None: the representation supporting task performance is either not available in the task space, either not visible, or not observable (or a combination of them)

3.b Other than:

3.b.1 Less information than required is provided by the considered representation.

3.b.2 More information than required is provided by the considered representation.

3.b.3 Different information than required may be available.

3.c Wrong timing: the resources required is available, but either

3.c.1 Later than required, or

3.c.2 Earlier than required

The results of such analysis may be stored in a table with the following information:

- Task: the activity currently analysed, together with some properties relevant to our analysis;

- Representation distribution: the resources supporting task performance and their distribution;

- Guideword: the type of interaction failure considered;

- Explanation: how an interaction failure has been interpreted for that task;

- Causes: the potential causes for the interaction failure considered and which configuration of resources might have generated the problem;

- Consequences: the possible effects of the occurrence of the interaction failure in the system; 
- Recommendation: suggestions for an alternative (if any) distribution of resources, able to better cope with the considered interaction failure.

Thus, our analysis aims at identifying better representations (or distributions of them) that could be more suitable for carrying out the considered tasks. Not only the evaluation has to consider if a different allocation of resources may be envisaged but also if different representations of information -which could involve considering different devices- may result in a significant improvement for the overall system's safety and usability.

\section{THE CASE STUDY}

In order to show potential application of our method we consider a case study related to a real setting: the Rome-Ciampino ACC (Area Control Centre). We visited the centre and interviewed a number of controllers working in it. It hosts a number of en-route controllers' teams plus an approach working position in charge of controlling and sequencing the aircraft access (e.g.: landing) to the runways of the close major Fiumicino airport. As far as the en-route working position is concerned, the airspace is partitioned, by horizontal and vertical divisions, into a number of geographical regions known as sectors. Aircraft in an en route sector are managed by two controllers working closely, but having individually different roles and concerns: the executive controller maintains continuous contact with aircraft using the VHF radio and headphones and is directly responsible for maintaining the appropriate separation distance between aircraft; the strategic or planning controller basically performs mediumlong term planning (identify future conflicts, planning future traffic), updates the system and decides how to separate flights also co-ordinating with strategic controllers of adjacent sectors.

As Figure 1 shows, the en-route position is equipped with five screens on which not only the aircraft are visualised (colourful and interactive flight labels are used to manage the different planes in the sector), but also electronic information (flight strips, monitoring data, sector boundaries, airways, etc.) is displayed.

If, on the one hand, the activities performed in the en-route working position make effective the paper-stripless environment available in Ciampino ACC centre, on the other hand this situation is different for the approach working position, where strict time constraints require an even quicker interaction between the different controllers working in the same position and a fully real-time awareness of the current ongoing activities, which can be reached only by paper strips that enable rapid annotations, and 
facilitates information sharing (the paper strip rack is generally located inbetween the two controllers).

Apart from other roles (a chief controller, a technician supervisor, one flow controller), there are also three (or more) supervisors that receive various types of alarms and have also the responsibilities of making decision about closing/opening sectors (usually dynamically divided in a vertical manner), depending on data about the estimated traffic size and the airport capacity, and also handling personnel resources. They can be regarded as the only role without any "dedicated" position within the control room.

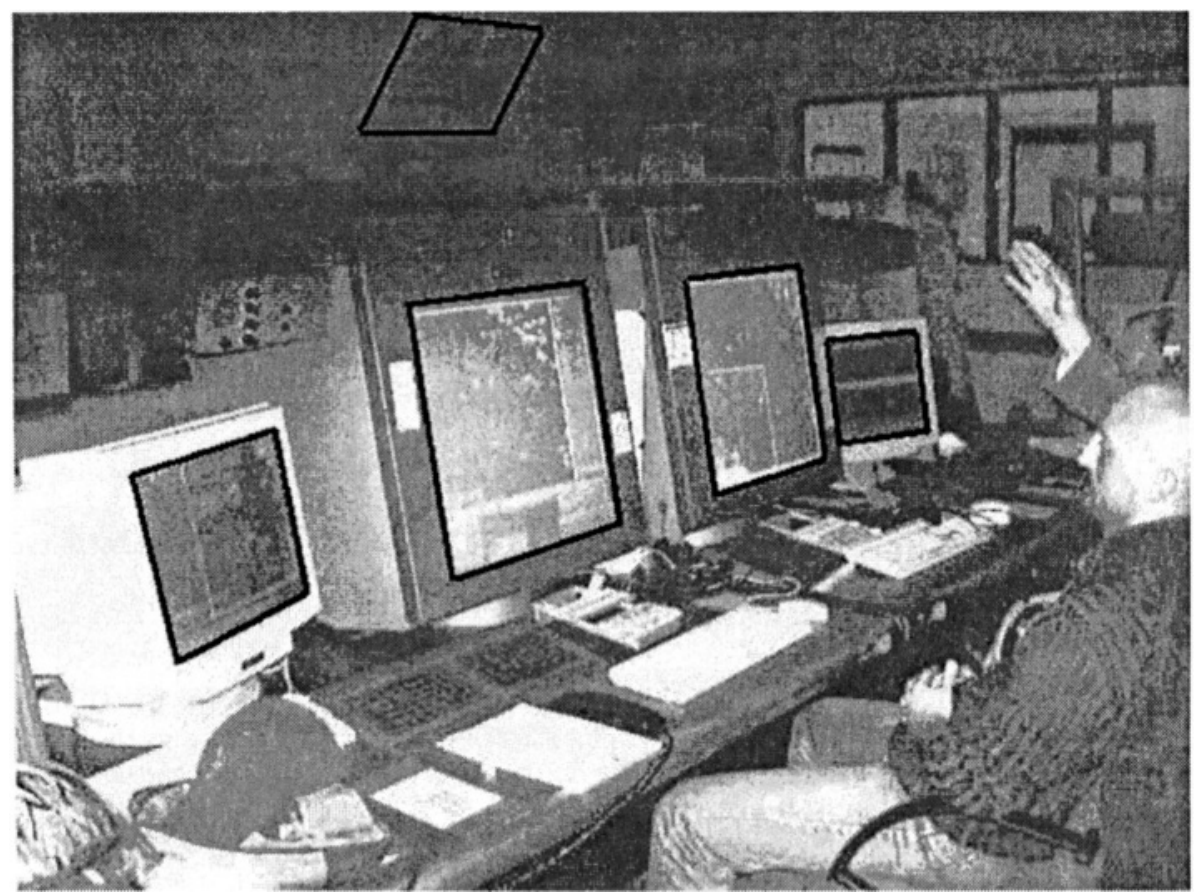

Figure 2. En-route position at the Air Traffic Control Centre in Ciampino.

\section{THE METHOD APPLICATION TO THE CASE STUDY}

We consider two controllers' activities related to the Ciampino case study in order to show how to apply our method. 


\subsection{Take over position between two controller teams (approach working position)}

\subsubsection{Description of the scenario}

During the working day there is a number of shifts and handovers of positions (when a different team of controllers prepares to take-over responsibility of the sector) within the control room, since no more than two hours is recommended in front of a radar screen. For example, in the Approach working position -the busiest in the control room and the only one in which paper strips are still used- the handover requires from the new team to build the mental picture and reconstruct the traffic events in the previous working period by accessing the traffic context (e.g.: observing a few minutes the air traffic, asking questions, hearing communications between controllers in charge, ...) and looking at the paper strips (which provide them with the history of the clearances given to the pilots and useful hints to derive the current strategies used for resolving conflicts and expedite traffic). However, especially during peak hours and in situation of high traffic, the need of taking over a position by giving verbal reports to the new team could be less than desirable as it might strongly interfere with controllers' activity because of interruptions, distractions and interferences of acoustical information.

\subsubsection{Motivations for the chosen scenario}

In the envisioned system, a possible solution to such interferences is providing (during the 'overlapping' period) the new team with a PDA, a device suitable for storing history and context, allowing them to reconstruct the current situation (while they are still able to hear communications in the room) without disturbing too much the operative team. In this way the need for verbal communications among the two teams (e.g.: verbal reporting from the controllers in charge, request of clarification from the new team, etc.) for taking over a position should be reduced to a minimum.

\subsubsection{Description of the task model}

In the task model shown in Figure 2 we analyse the issues involved during such 'teamwork overlapping' period and the consequent take over. Who is going to become in short time the 'new' controller has to understand what is currently going on by gathering information from the paper strips and from other information sources available (PDA, vocal information, ..) so as to continuously build/update the mental picture of the situation until 
actually "take over", which means replacing the other controller and start to fully control the ATC equipment.

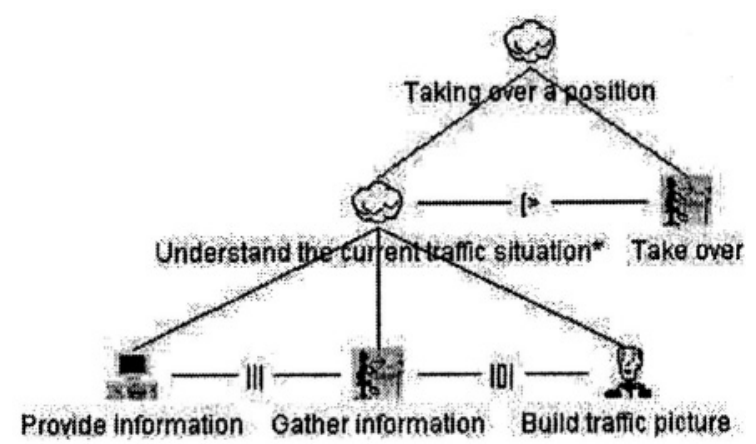

Figure 2. The task Taking over a position.

If we consider the parent task as a whole, diverse representations come into play: before actually taking over there are paper strips and vocal communications exchanged in the close proximity of the suite (which are globally shared by all the teams); information on the PDA (which is visible only to the non-operative controllers who will manage the traffic in the next future). After taking over, the PDA is supposed not to be available anymore to the operative controller who can access to a plenty of information through the screens and the headphones. One of the most interesting issues raised by the scenario is the switch between the different roles (and consequent different work contexts in terms of tools, media and platforms used) that the same person has to carry out when moving from non-operative to operative role.

Role: Approach controller taking over a position

Task: Taking over a position (replace the controller in charge and start to control the system)

1. Analysis of the task and related properties

1.a Task category: abstract task

1.b Task platforms: PDA, paper strips, screens (with related tools), telephone, headphone, cognitive internal information gathered during overlapping period (by hearing communications, interacting with their PDA, and eventually asking questions to controllers in charge).

1.c Task frequency: medium

1.d Other properties (that could affect task 'criticality'):

Need of starting immediately to operate in the real system (e.g.: respond

to pilots) as soon as take over is completed.

2. Analysis of the representations associated with the task. 
The representations supporting the task are: i)Mental picture of the current traffic situation (built within the overlapping period by hearing communications in the ATC environment, interacting with the PDA, asking questions to controllers in charge, etc.); ii)Graphical representation on the screens (distance might prevent the non-operative team to access all the data); iii)Audio data coming from headphones.

2. a Availability: both external representations (graphical: on the screens; audio: from headphones) and internal representation (mental picture) of ATC system.

2.b Accessibility: concurrent access to audio and graphical information (audio/graphical data continuously compared to the information maintained in controller's mental picture). Sequential access to verbal data from headphones.

2.c Mobility: the users of the PDA are supposed to hear other colleagues' conversations while using PDA: then, they should not need to move for accessing information, albeit they could

2.d Perceptibility: Data from headphones and PDA: Local; Information on screens: Shared by team members

2.e Persistence: transient access to audio information;

2.fFlexibility of cognitive tracing and interactivity: high (full control of the system).

2.g Operations and actions supported

2.g.1 Comparability with other objects/representations available in the task space: Low. Representations on the screens not immediately comparable to that they received on the PDA (used during the overlapping period), because of diversities between the supporting platforms;

2.g.2 Combinability (should allow user to select novel forms of combinations of information): Low. Controllers have to switch from using PDA to using huge screens; no combination is allowed.

2.g.3 Ease of production: Low. Every personalisation allowed on the PDA (personal device, with limited capabilities and no possibility to update the real system) is banned on the huge screens operating in the real system (which are shared by different teams, and have plenty of interaction techniques to modify the system), in order to prevent the new team to have difficulties in interpreting views resulting from adaptation processes.

\section{Analysing deviations from the task plan}

3. a Guideword: Other than (when talcing over, the information provided to controllers is different from that expected)

3.a. 1 Less: the information provided is less than required

Causes: controllers have annotated some information on their PDA, and this device is supposed not to be used anymore in the fully operative system. 
Consequences: possible overload on the memory of the controllers, who tries to recall their annotations and possible time wastage for rebuilding such information; possible distractions for the controller.

Recommendations: enable controllers to have quickly available their annotations (also providing automatic deletion of 'obsolete' information) in the real system

3.a. 2 More: the information provided is more than expected

Causes: the fully operative system has to provide information on every aircraft $(\mathrm{a} / \mathrm{c})$ controlled, whereas during the overlapping period the controllers had put their attention just on some selected a/c (those with 'highest priority', according to some priority criteria)

Consequences: controller wastes time identifying concerned $\mathrm{a} / \mathrm{c}$

Recommendations: enable the controller to e.g. select an a/c on the PDA and make it highlighted on the huge screen (improve combinability) and comparability (easiness in making associations between the two views)

3.a.3 Different: the information provided is different from expected

Causes: the representations provided by the PDA in the overlapping period might have slightly biased the controller's mental picture against the representations available in the fully operative system with huge screens and headphones (the representations may strongly differ), if the two representations are not immediately comparable.

Consequences: overload on the controllers' memory (they try to suit their mental picture to the current situation as it is represented in the fully operative system)

Recommendations: provide mechanisms to "smoothly" move from one device/picture to another, fully exploiting any possible cognitive progress the controller might have performed in the overlapping period (in anticipating future actions, decision making, strategy planning, etc.); consider the possibility of actively transferring information from PDA to real system (controllers should be enabled to easily make the correspondences between the different views: comparability) plus the possibility of using PDA as a control device for the real system -although for a short period of time (combinability).

The recommendations highlight under which conditions the use of a mobile device can provide useful support in the context considered.

\subsection{De-combining air traffic sectors}

\subsubsection{Description of the scenario}

In a situation of critical meteorological conditions, the Control Center supervisor manages information about the upcoming traffic in order to 
trigger de-combining of two sectors. He is also monitoring the situation of the control working positions in order to re-distribute the controllers' workload across the control room. The ATC supervisor has the responsibility of making decision about closing/opening sectors (usually dynamically divided in a vertical manner), depending on data about the estimated traffic size and the airport capacity, and also personnel resources available on site. The supervisor is permanently contacted from various fixed working positions in the control room, receive various types of alarms, and need to base his decisions on integrating various sources of information.

\subsubsection{Motivations for the chosen scenario}

ATC supervisor can be regarded as the only role without any "dedicated" position within the control room. The need of having permanent access to real time traffic information may imply a high level of mobility of the supervisor in the control room. In this case, our hypotheses is that a mobile device, as a PDA, could offer a valuable support in carrying out his tasks.

\subsubsection{Description of the task model}

In order to de-combine two air space sectors, the supervisor has to identify overloaded air traffic sectors, and the level of criticality of the upcoming air traffic; at the same time, he has to evaluate the on-site workload allocation of the controllers, and also to identify available personnel for taking up the control of a new sector. The complex information supporting supervisor's task is available from several sources distributed in the task space: flight information system, air traffic monitoring system, radar, flight progress strips, meteorological information, etc. The graphical representation of the task considered is presented in Figure 3.

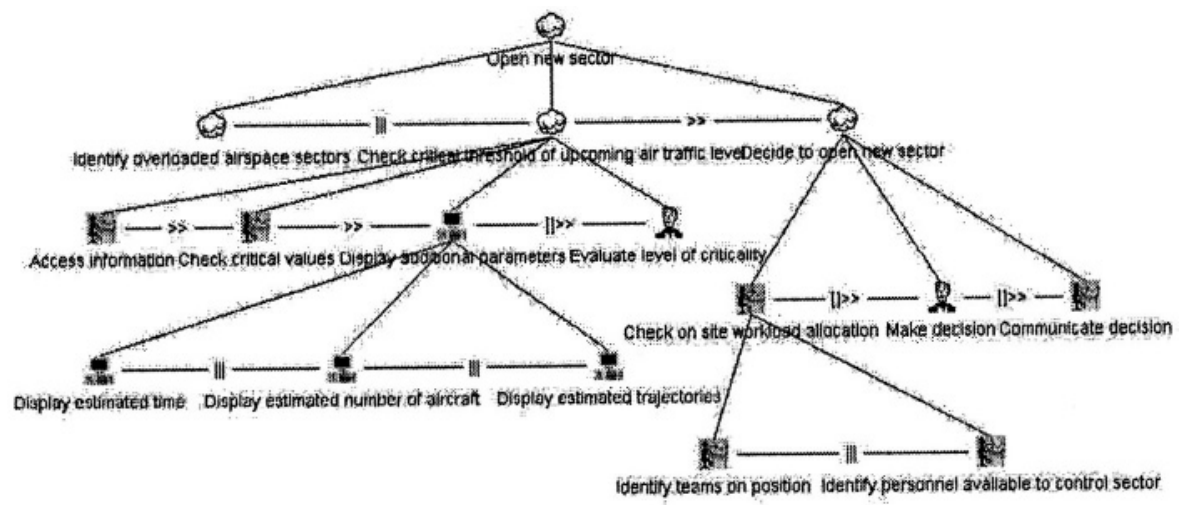

Figure 3. The "open new sector" task. 
In the followings, we will look in more details at one of the subtasks identified in the above model, and reason about the possibilities of introducing a mobile device in order to support the considered task.

Role: Control Centre supervisor Task: Open new sector

SubTask: Check critical threshold of the upcoming air traffic level

1. Analysis of the task and relatedproperties

1.a Task category: abstract task

1.b Task platforms: an integrated set of tools (computer displays, telephones, paper-based documentation) is available at the level of the dedicated working position in order to allow the access to radar information, flight information system, flight progress strips, meteorological information, etc.; alternatively, a mobile platform, i.e. PDA, could support the access to (some of) the required information.

\section{1.c Task frequency: high}

1.d. Other properties impacting on task 'criticality': they are, for instance, the need for real time access to information about the current and estimated up-coming air traffic level; strong dependency with the consequent task (decision to open a new sector will be further based on the information manipulated by this task).

2. Analysis of the representations associated with the task

Several information objects supporting task performance may be identified: normal and critical threshold of upcoming air traffic level, additional parameters as estimated numbers of aircraft together with time intervals planned trajectories, etc.

2.a Availability: they are available in both graphical and numerical representation forms.

2.b Accessibility: the representation forms and media (in this case, large computer screens) allow user a concurrent, easy access to a variety of information. If accessing the same information with a PDA, it is expected that its physical constraints (i.e. screen size) will make sequential the access to information, therefore increasing the time and effort needed to visualise the same items. On the other hand, a PDA would allow a permanent access to the required information, even if user changes his position across the control room.

2.c. Mobility: The user is expected to move in the control room for accessing the needed information.

2.d. Observability: similarly, using a small screen device is likely to change the observability of information, from being easily shared with other members of the team, to locally available to the user of the device.

2.e Persistence: critical information (e.g.: threshold of the upcoming air traffic level) is graphically represented, so allowing a non-transient access. 
2.f Flexibility of cognitive tracing and interactivity. In the considered case, the parameters of interest are changing autonomously, in accordance with the real-time situation of the air traffic flow. Controllers have no or minimal permission to operate changes, to annotate or update an external electronic representation. A standard working position will be equipped with no input device (i.e., a keyboard), being allowed only direct manipulation of the objects already available on the screen.

2.g Operations and actions supported

2.g.1 Comparability. the graphical representation of information employed (i.e., clustered columns ) gives user the possibility to directly compare various values of the monitored variables (i.e., by rapidly perceiving differences between the high of two columns).

2.g.2, 2.g.3 Combinability - possibility to combine and reconfigure or re-represent the information of interest: highly supported in the current ATC work settings. For instance, the information contained in an electronic flight strip can be displayed in two different formats; the values of the upcoming traffic may be represented graphically as well as numerically, etc. For the hypothetical situation of using a PDA, the question is how to effectively display the relevant information in the perimeter of a very small screen space, while maintaining a high level of interactivity. For instance, a solution would be to reduce the amount of graphics, rely mainly on the numerical representation of information, and using additional codes (i.e. sounds) in order to facilitate user's rapid discrimination of critical information.

Analysing deviations from the task plan.

By applying the proposed guidewords to some of the properties described above, it is possible to identify potential deviations from the task plan. For instance:

3. a Guideword: None (the property of the representation of interest is not available)

Information not visible.

Causes: difficulties in perceiving the relevant information due to some ergonomic issues: the object represented is too small, ambiguous shape, wrong choice of colour, etc.; but also supervisor' s distraction / interruption by other activities, etc.

Representation not persistent.

Causes: Rapid change of information values; does not allow the time user needs to internalise the perceived information and to integrate it with the other information supporting his decision making. 
Consequences: if there is no information available in the task space (not visible, not persistent) then, various types of task failure can occur (i.e., stop task performance, delay, etc.).

Recommendation. Rely on multiple ways of representing the same information (i.e., visual and auditory): access to concurrent representation of the same information could be especially important for users 'on the move', who allocate their attention on several competing tasks.

Design should facilitate a rapid perception of the relevant information, and support an accurate interpretation of its signification (i.e., estimation of the air traffic flow - under/ over a critical level, or its approximate value). For instance, the discrimination of the critical information could be facilitated by adequate use of colour, use of multimedia facilities as animation, blinking images, the use of sound, etc.

\section{CONCLUSIONS}

Some authors have criticised task-based models of interaction, claiming that such approaches are not able to address the importance of context in interaction, and the distinction between tasks as described and task as observed in practice. A key concern in this case is the problem of characterising the context of action.

Distributed cognition is particularly concerned with the context of work and the notion of distributed representational state, and the importance of mutual knowledge in guiding action. However, DC analysis is criticised for its high qualitative approach and difficulties of translating its results in the design practice.

An integration of the two approaches could be beneficial, producing a mutual reinforcement at their both conceptual and methodological level. In this paper we have presented a method aiming at achieving such a goal. The method has been discussed within a case study in the air traffic control domain.

Future work will be dedicated to further apply the method presented in this paper to the design of interactive safety-critical systems exploiting the use of mobile devices.

\section{ACKNOWLEDGEMENTS}

This work has been supported by the EU TMR ADVISES (http://www.dcs.gla.ac.uk/advises). We also thank ENAV for allowing us to access the Ciampino ATC Centre. 


\section{REFERENCES}

Buisson, M., Yannick Jestin, Design issues in distributed interaction supporting tools: mobile devices in an ATC working position, Mobile HCI 2001.

Fields, R.E., Wright, P.C., Marti, P. \& Palmonari, M. (1998). Air traffic control as a distributed cognitive system: a study of external representation. Proceedings of the $9^{\text {th }}$ European Conference on Cognitive Ergonomics - ECCE-9, EACE Press.

Fields, R., Paternò, F., Santoro, C., Tahmassebi, S.(1999). A method for the comparison of design options for allocating communication media in a cooperative and safety-critical context. ACM Transactions in Computer-Human Interaction Vol.6, N.4, pp.370-398, ACM Press, December 1999.

Hollan, J., Hutchins, E. \& Kirsch, D. (2000). Distributed Cognition: Toward a New Foundation for Human-Computer Interaction Research, in ACM Transactions on Computer-Human Interaction, 7 (2), p. 174-196.

Hutchins, E. (1995) How a cockpit remembers its speeds. Cognitive Science, 19, pp. 265-288.

McDonough (2002), Boeing Addresses Air Safety with Wireless 'Flight Bag'. Wireless NewsFactor. http://wireless.newsfactor.com/story.xhtml?story_id=18486

Mertz, C., Chatty, S. and Vinot, J.-L.. The influence of design techniques on user interfaces: the DigiStrips experiment for air traffic control. In Proceedings of HCI Aero IFIP 13.5, 2000.

Mori, G., F. Paternò, C. Santoro (2002). CTTE: Support for Developing and Analyzing Task Models for Interactive System Design. IEEE Transactions on Software Engineering, pp. 797-813, August 2002 (Vol. 28, No. 8).

Norman, D. A. (1988). The psychology of everyday things, Harper Collins: Basic Books.

Paternò, F, Santoro, C. (2002). Preventing user errors by systematic analysis of deviations from the system task model. International Journal Human-Computer Studies, Elsevier Science, Vol.56, N.2, pp. 225-245.

Paternò, F. (2003). ConcurTaskTrees: an engineered notation for task models. In Dan Diaper \& Neville A.Stanton (Eds.), The Handbook of Task Analysis for Human Computer Interaction. London: Lawrence Erlbaum Associates, pp.483-503. 